\title{
Abstracts
}

\section{Genetics and Epidemiology}

\author{
Population-based Rates of Urinary Tract Infection \\ Hospitalization by Spina Bifida Status in Tennessee \\ Medicaid, 2005-2013 \\ Gebretsadik, Tebeb ${ }^{1}$, Cooper, William O. ${ }^{1}$, Ouyang, \\ Lijing $^{2}$, Thibadeau, Judy ${ }^{2}$, Tesfaye, Sarah ${ }^{1}$, Mitchel, \\ Ed $^{1}$, Carroll, Kecia N. ${ }^{1}$ \\ ${ }^{1}$ Vanderbilt University Medical Center \\ ${ }^{2} C D C$
}

Background Individuals with Spina Bifida are at increased risk of urinary tract infections (UTI). We examined population-based rates and rate ratios of UTI hospitalizations in individuals up to age 65 years by Spina Bifida status.

Methods We conducted a population-based, retrospective cohort study including individuals continuously enrolled in Tennessee Medicaid and who lived in one of the Emerging Infections Program's Active Bacterial Surveillance counties in Tennessee, 2005-2013. Females included were non-pregnant. Spina Bifida diagnoses were captured using vital records and International Classification of Diseases, Ninth Revision (ICD9) codes. UTI hospitalizations were identified from ICD-9 codes in 1) any of the diagnosis fields or 2) in the $1^{\text {st }}$ or $2^{\text {nd }}$ position (primary UTI). We calculated the yearly rates of subjects hospitalized with a UTI over the persons eligible. We used Poisson regression to estimate rate ratios to compare yearly UTI hospitalization rates between individuals with and without SB.

Results Over the 9 year period, 1,239,362 individuals were included, and 2493 met criteria for Spina Bifida. In the non-Spina Bifida group, UTI hospitalization rates ranged from 8.5 to $10.6 / 1000$ within study years. Rates were higher in individuals with Spina Bifida ranging from 59 to $77.9 / 1000$ with an average rate ratio of 7.5 (95\% CI 7.12, 7.94) compared to those without Spina Bifida during the study period. Individuals with Spina Bifida had primary UTI hospitalizations rates between 35.7 to $58.1 / 1000$, with an average rate ratio of $8.6(95 \% \mathrm{CI} 7.93,9.30)$ compared to those without Spina Bifida.

Conclusions In Tennessee Medicaid, individuals with Spina Bifida have significantly increased rates of UTI hospitalizations compared to those without Spina Bifida. Future work will investigate culture confirmed UTI hospitalizations based on medical records.

\author{
The Inspiring Tale of Infant Survival of Spina \\ Bifida, a Historical Perspective \\ Pace, Nelson ${ }^{1}$, Wilde, David J. ${ }^{2}$ \\ ${ }^{1}$ University of North Carolina at Chapel Hill \\ ${ }^{2}$ University of Texas Health Sciences Center at San An- \\ tonio
}

Background Over the past century, infant mortality, death of a liveborn child in the first year of life, has decreased in the US from 10 to $0.6 \%$, a $94 \%$ reduction in infant mortality. Great efforts to prevent and treat disease have made this possible. Among the monumental advances in perinatal clinical care in last 50 years, has been the increased survival of infants born with a birth defect. Advancements in treatment and clinical decision- making have made some birth defects, like Spina Bifida, a congenital anomaly that rarely results in death. Spina Bifida occurs in 1 out of every 3000 live born infants in the US.

Methods We reviewed studies on survival among infants with Spina Bifida to present synthesized results of infant survival juxtaposed with advancements in clinical care.

Results Whether or not to even treat an infant with Spina Bifida or when to treat was debated for several decades. Prior to the 1960 s, only $11-30 \%$ of untreated infants with Spina Bifida survived beyond 6 months while $24-41 \%$ of treated cases survived beyond 1 year. Use of shunts to treat and prevent hydrocephalus paralleled survival rising to $60 \%$. Improved shunts and an- 
tibiotic use (1970s) led to 60-86\% survival. Survival of Spina Bifida has gradually improved to be currently between $92-97 \%$ due to antenatal detection and monitoring and improvements in surgery and post-operative care (1980s). Shunts and clinical guidelines to preform treatment soon after birth have likely resulted in the largest improvement in survival.

Conclusions Spina Bifida is an inspiring model for how other birth defects or clinical conditions can also have improvements in survival through technological advancements and changes in clinical guidelines.

\section{Surveillance Survey of Family History in Children with Neural Tube Defects \\ Dupepe, Esther, Patel, Daxa, Rocque, Brandon G., Hopson, Betsy D., Arynchyna, Anastasia, Bishop, \\ E R., Blount, Jeffrey \\ University of Alabama at Birmingham}

Background Although there are known risk factors for the development of neural tube defects, little data exists on the role of family history. The goal of this study is to describe the family history in our population of patients with neural tube defects.

Methods Surveys were completed for 254 patients who were accompanied by their biological mother during their annual visit to the multi-disciplinary Spina Bifida Clinic. All patients seen in this clinic have a diagnosis of neural tube defect (e.g. myelomeningocele, lipomeningocele, split cord, congenital dermal sinus, etc). Each mother answered questions regarding known neural tube defect risk factors, their pregnancy and as well as the family history of neural tube defects, other nervous system disorders and birth defects.

Results The overall prevalence of family history of neural tube defects in children with neural tube defect was $16.9 \%(n=43)$, of which $3.1 \%(n=8)$ were in first-degree relatives. In patients with myelomeningocele, $17.7 \%(n=37)$ had a positive family history for neural tube defects with $3.8 \%$ in first-degree relatives. Family history in the paternal lineage was $8.7 \%$ versus $10.6 \%$ maternal. Twenty-two $(8.7 \%)$ had family history of other congenital nervous system disorders. Fifteen $(5.9 \%)$ had family history of Down syndrome, twelve $(4.7 \%)$ had family history of cerebral palsy, and thirteen $(5.1 \%)$ patients had family history of clubfoot. Fourteen $(5.5 \%)$ had family history of cardiac defect and $13(5.1 \%)$ had family history of cleft lip or palate. Conclusions The family history of neural tube defect was $16.9 \%$ in children with neural tube defect with- out a difference in maternal and paternal lineage. This high rate of positive family history suggests that genetics and epigenetics may play a larger role in the pathogenesis of neural tube defect in the modern era of widespread folate supplementation.

\section{Longitudinal Study of Bladder and Bowel Conti- nence among Spina Bifida (SB) Patients in the National Spina Bifida Patient Registry (NSBPR)}

Liu, Tiebin ${ }^{1}$, Ouyang, Lijing ${ }^{1}$, Thibadeau, Judy ${ }^{1}$, Wiener, John S. ${ }^{2}$, Walker, William ${ }^{3}$, Routh, Jonathan C. ${ }^{2}$, Smith, Kathryn ${ }^{4}$, Castillo, Heidi ${ }^{5}$, Castillo, Jonathan $^{5}$, Van Speybroeck, Alexander ${ }^{4}$, Clayton, Gerald H. ${ }^{6}$, Valdez, Rodolfo A. ${ }^{1}$

${ }^{1} C D C$

${ }^{2}$ Duke University Medical Center

${ }^{3}$ University of Washington

${ }^{4}$ Children's Hospital Los Angeles

${ }^{5}$ Texas Children's Hospital

${ }^{6}$ Children's Hospital Colorado, Aurora

Background Incontinence associated with neurogenic bowel and bladder is common among individuals with SB and achieving continence for them is a lifetime management goal. We investigated changes in urinary and bowel continence status over time and factors associated with this status among individuals with SB.

Methods We used data from the NSBPR from 2009 through 2015. For this study, we excluded all visit data collected from children when they were under 5 years of age. To account for repeated measurements in the same individual over time, we used generalized estimating equation (GEE) models to analyze factors associated with continence status.

Results The study cohort consisted of 5340 individuals with SB who contributed 13062 annual clinic visit records during the study period. At follow-up, the average age was 16.7 years and $22.5 \% / 21.1 \%$ of the patients had undergone bladder/bowel continence surgery, and $92.7 \% / 63.7 \%$ of the individuals used some form of bladder/bowel management. About $27.5 \% / 32.5 \%$ of the patients experienced changes in bladder/bowel continence status. At the most recent visit, $44.4 \% / 51.3 \%$ reported bladder/bowel continence. In multiple regression models, both bladder and bowel continence were significantly associated with older age, female, non-Myelomeningocele diagnosis, history of continence surgery, lower level of lesion, higher mobility level, and private insurance. Both bladder and bowel management were also associated with 
continence independent of all other factors (adjusted ORs and 95\% CI: 3.85, 3.22-4.62; 1.10, 1.01-1.20, respectively).

Conclusions Multiple factors are significantly and independently associated with bladder and bowel continence status among SB patients. The association of surgical and management approaches with bladder and bowel continence was independent of demographic, social, and other medical-related factors.

\section{Age Distribution of Surgical Procedures and Health Outcomes in the National Spina Bifida Patient Reg- istry (NSBPR)}

Alabi, Noreen ${ }^{1}$, Thibadeau, Judy ${ }^{1}$, Wiener, John S. ${ }^{2}$, Conklin, Michael ${ }^{3}$, Dias, Mark S. ${ }^{4}$, Sawin, Kathleen J. $^{5}$, Valdez, Rodolfo A. ${ }^{1}$

${ }^{1}$ Centers for Disease Control and Prevention

${ }^{2}$ Duke University Medical Center

${ }^{3}$ University of Alabama Medical Center

${ }^{4}$ Penn State University

${ }^{5}$ Children's Hospital of Wisconsin

Background Spina Bifida (SB) may cause urine and stool incontinence, cognitive impairments, loss of ambulation, and pressure sores. The management of these conditions commonly involves surgical procedures. We examined age distributions of selected conditions and procedures among members of the NSBPR.

Methods The NSBPR (2009-2013) includes 4,664 patients and all had data on key health outcomes. A subgroup of 3,801 patients had data on selected surgical procedures. Relative frequency was calculated for 22 age groups $(<1 \mathrm{y}$ to $\geqslant 25 \mathrm{y})$.

Results Respectively, 3,159 patients with a myelomeningocele (MMC) and 642 with a non-myelomeningocele (NMMC) diagnosis reported totals of 17,265 and 1,626 surgical procedures in their lifetime. The median age by procedure was: neurologic $(<1 \mathrm{y}$ for both MMC and NMMC); orthopedic (5 y for MMC and $8 \mathrm{y}$ for NMMC); gastrointestinal (9 y for MMC and $3 \mathrm{y}$ for NMMC); urinary ( $8 \mathrm{y}$ for MMC and $5 \mathrm{y}$ for NMMC); skin (11 y for both MMC and NMMC); and other ( $2 \mathrm{y}$ for MMC and $3 \mathrm{y}$ for NMMC). For MMC cases, the respective ages with the lowest and highest prevalence of stool continence were 5 y $(22.1 \%)$ and $17 \mathrm{y}(49.1 \%)$. The equivalent values for NMMC cases were 6 y $(50.6 \%)$ and 19 y (70.6\%). Similarly, for MMC cases, the respective ages with the lowest and highest prevalence of urine continence were $5 \mathrm{y}$ $(20.0 \%)$ and 19 y (48.9\%). The equivalent values for
NMMC cases were 6 y (49.4\%) and 17 y (69.5\%). The prevalence of pressure sores was lowest at age $<1$ y (3\% MMC, 0\% NMMC) and highest at age 17 y $(30.0 \%)$ for MMC and age 20-25 y (20\%) for NMMC cases. The prevalence of full-time wheelchair users among MMC cases ranged from $18 \%$ (at age 5 y) to $54 \%$ (at age $\geqslant 25 \mathrm{y}$ ).

Conclusions The distributions of surgical procedures and health outcomes vary widely by age among patients with SB. At all ages, MMC cases showed a higher prevalence of less favorable health outcomes than NMMC cases.

\section{Estimating the Economic Impact of Spina Bifida in the United States \\ Inouye, Brian M. ${ }^{1}$, Wang, Hsin-Hsiao ${ }^{1}$, Jiang, Ruiyang $^{1}$, Tejwani, Rohit ${ }^{2}$, Wiener, John S. ${ }^{1}$, Purves, J T. ${ }^{1}$, Routh, Jonathan C. ${ }^{1}$ \\ ${ }^{1}$ Duke University Medical Center \\ ${ }^{2}$ Duke University School of Medicine}

Background An increasingly large number of children with Spina Bifida (SB) are surviving beyond infancy into adolescence as a result of modern medical and surgical advances. However, little data exists regarding the economic implications of modern SB care. We examined the charge data in national databases to provide annual nationwide estimates.

Methods We analyzed the 2006-2010 Nationwide Inpatient Sample (NIS) and Nationwide Emergency Department Sample (NEDS). We used ICD-9-CM codes to identify SB patients. We abstracted demographic and charge data from each database. The charges were estimated by multiple imputation method.

Results In total, SB patients had 37,584 inpatient admissions (33.5\% males) and 49,200 ER encounters (27.1\% males), identified by NIS and NEDS respectively. Of the ER visits, 17,699 (36.0\%) patients were subsequently admitted. The majority of patients were adults (66.5 in NIS, $72.9 \%$ in NEDS) with mean age of $28.4( \pm 1.2)$ and $29.2( \pm 1.2)$ years, respectively. Most patients had public insurance in both NIS (64.3\%) and NEDS (65.9\%), and the South was the most common geographic region (NIS 39.5\%, NEDS 36.6\%). The total estimated inpatient charges for all ages were $\$ 1.670$ billion/year. Mean ER charges for all patients captured by NEDS were $\$ 1,555 /$ encounter for a total of \$120 million/year. A 2006-2010 trend analysis demonstrated an increasing charge per inpatient admission $(\mathrm{RR}=1.05, p=0.003)$ and $\mathrm{ER}$ visit $(\mathrm{RR}=1.09$, $p<0.001)$. 
Conclusions Based on data from inpatient and ER sources, the economic impact of SB was at least $\$ 1.79$ billion in 2010 alone. This figure is likely a significant under-estimate of the true economic burden of SB, as it accounts for neither non-ER outpatient SB management (such as outpatient visits, medications, and surgeries) nor societal costs such as patient and caregiver time away from work and/or school.

\section{Lifetime Costs of Care for Infants Born with Spina Bifida in the United States}

Grosse, Scott ${ }^{1}$, Ouyang, Lijing ${ }^{1}$, Waitzman, Norm ${ }^{2}$, Tilford, J. M. ${ }^{3}$, Do, ThuyQuynh ${ }^{1}$, Thibadeau, Judy ${ }^{1}$

${ }^{1} C D C$

${ }^{2}$ University of Utah

${ }^{3}$ University of Arkansas for Medical Sciences

Background Individuals with Spina Bifida (SB) face higher medical and non-medical costs. Estimates of lifetime costs can be used to understand the resources needed to provide care and also to quantify potential cost savings from primary prevention policies, such as folic acid fortification.

Methods A synthetic cohort of infants born with SB was created by multiplying age-specific incremental survival probabilities and incremental annual costs for individuals with $\mathrm{SB}$ relative to individuals in the general population. Medical costs were derived from two published studies, one conducted using data from California in the late 1980s on children with SB and one using US health insurance claims data from the early 2000s on children and adults living with SB. National estimates of per-child costs of special education and developmental services were adjusted from California data. Estimates of parental caregiving time costs were based on Arkansas survey data. Costs were calculated for future years using a 3\% annual discount rate. Results The mean discounted lifetime cost per infant with SB is estimated to be $\$ 791,900$ in 2014 US dollars, or $\$ 577,000$ after excluding caregiving costs.[1] The present value of incremental medical expenditures alone is $\$ 513,500$.

[1] Grosse, S., et al., Retrospective assessment of cost savings from prevention. American Journal of Preventive Medicine, 2016, 50(5): p. S74-S80.

Conclusions Cost estimates are important for assessing resource needs and projecting cost savings from primary prevention strategies. Because cost estimates are not directly transferable across countries, analyses are needed for other countries. Also, to inform disease prevention and health promotion efforts, it would be useful to understand how lifetime costs vary based on SB characteristics, such as spinal lesion level and the co-occurrence of other malformations. 